Pacific Journal of Mathematics

THE EXTENDABILITY OF SOLUTIONS OF PERTURBED 


\title{
THE EXTENDABILITY OF SOLUTIONS OF PERTURBED SCALAR DIFFERENTIAL EQUATIONS
}

\author{
STEPHEN R. BERNFELD
}

\begin{abstract}
Much research has been concerned with the preservation of particular properties of solutions for the perturbed differential equation. In particular many results have been obtained for the perturbation of either stable or bounded differential equations. However, the preservation under perturbation of a more fundamental property of solutions; namely, extendability of solutions on $\left[t_{0}, \infty\right)$, has not been fully explored, and it is this problem which this paper is concerned with.
\end{abstract}

Particular sufficient conditions on the behavior of a class of perturbing scalar functions $\{\phi(x)\}$ are obtained to insure that solutions of

$$
\dot{x}=\gamma(x)+\phi(x)
$$

exist in the future whenever solutions of

$$
\dot{x}=\gamma(x)
$$

exist in the future. It is shown, moreover, that for a rather broad class of unperturbed scalar differential equations the admissible perturbing functions $\{\phi(x)\}$ are dependent on the unperturbed functions $\{\gamma(x)\}$. This does not often occur in the case of stability and boundedness. However, under more restrictive conditions on the unperturbed equation, such as linearity, admissible classes of perturbing functions are provided which are independent of the unperturbed equation.

We obtain necessary and sufficient conditions on the unperturbed equation so that all solutions of a certain class of perturbed equations exist in the future.

The techniques are applied to obtain results on the uniqueness and boundedness of perturbed scalar differential equations.

The reader is referred to ([1], [3], [4], [6]) for a through treatment of the theory of the extendability of solutions of differential equations.

2. Notation and preliminaries. Let $R^{d}$ denote Euclidean $d$-space and $|\cdot|$ will denote any $d$-dimensional norm. We represent a solution of the differential equation

$$
\dot{x}=f(t, x)
$$

satisfying $x\left(t_{0}\right)=x_{0}$ by $x\left(t, t_{0}, x_{0}\right)$. 
Consider the following problem: assume all solutions of (E)

$$
\dot{x}=f(t, x),
$$

where $f: R \times R^{d} \rightarrow R^{d}$ is continuous, exist in the future; then for what class of functions $g(t, x)$ do all solutions of

$$
\dot{x}=f(t, x)+g(t, x) \text {. }
$$

In this discussion we will impose a growth condition on the solutions of $(\mathrm{E})$ by requiring the existence of a function $\phi:[0, \infty) \rightarrow$ $(0, \infty)$ such that

$$
|f(t, x)| \leqq \phi(|x|) \text { for all } t \in R \text {, and } \int^{\infty} \frac{d r}{\phi(r)}=\infty \text {. }
$$

This condition is sufficient for all solutions of $(\mathrm{E})$ to exist in the future (see [2 p. 30]). We say $\dot{\phi}(\cdot)$ is related to $f(t, x)$ if $(2.1)$ is satisfied.

Definition 2.1. Let $\Delta$ be the class of continuous functions $\phi$ : $[0, \infty) \rightarrow(0, \infty)$ and satisfying $\int^{\infty} d r / \phi(r)=\infty$.

Definition 2.2. Let $\bar{\Delta}$ be the class of those functions $\phi \in \Delta$ which are monotone increasing.

Because of the growth conditions imposed on $f$ we have essentially reduced the problem to studying the extendability of perturbed scalar differential equations. Namely, we shall analyze the following problem: Assume all solutions of

$$
\dot{r}=\gamma(r),
$$

$\gamma:[0, \infty) \rightarrow(0, \infty)$ is continuous, exist in the future; (it is not difficult to show that existence in the future is equivalent to $\int^{\infty} d r / \gamma(r)=\infty$ ) then for what class of functions $\phi(r)$ do all solutions of

$$
\dot{r}=\gamma(r)+\dot{\phi}(r),
$$

$\rho:[0, \infty) \rightarrow(0, \infty)$ exist in the future. Due to the theory of differential inequalities results for (ES) and (PS) imply corresponding results for (E) and $(\mathrm{P})$. Hence our discussion will in most part be confined to (ES) and (PS).

As pointed out before, one of the most fundamental questions concerned with perturbations is whether the perturbing term $\phi$ is independent of the particular function $\gamma$. In the following example we will show that the perturbation term must depend upon the unperturbed 
system in order to get meaningful results; namely, there exist solutions of (PS) that do not exist in the future when both $\gamma$ and $\phi \in \Delta$.

EXAMPle 2.3. Consider the scalar equation $\dot{x}=h(x)$ where $1 / h(x)$ is defined as follows: for each integer $n>0$ such that $n \leqq x \leqq n+1$,

$$
\begin{aligned}
& 1 / h(n)=n, 1 / h(n+1)=n+1, \\
& 1 / h(x)=1 / x^{2}, n+1 / n^{2} \leqq x \leqq n+1-1 /(n+1)^{2}, \\
& 1 / h(x) \text { is linear for } n \leqq x \leqq n+1 / n^{2} \text { and } \\
& \qquad(n+1)-1 /(n+1)^{2} \leqq x \leqq(n+1) .
\end{aligned}
$$

Hence from the construction of $h(x)$, we have for each $x_{0}>0$

$$
\int_{x_{0}}^{\infty} \frac{d x}{h(x)}>\int_{x_{0}}^{\infty} 1 / x^{2}+\sum_{n=\left[x_{0}+1\right]}^{\infty} \text { area } T_{n},
$$

where $T_{n}$ are the triangles whose sides are the graph of $1 / h(x)$. The area of $T_{n}$ is greater than $n / 2 \cdot 1 / n^{2}=1 / 2 n$. Therefore

$$
\sum_{n=\left[x_{0}+1\right]}^{\infty} \text { area } T_{n}=\infty \Longrightarrow \int^{\infty} \frac{d x}{h(x)}=\infty \Longrightarrow h(x) \in \Delta .
$$

We now consider the equation

$$
\dot{x}=h(x)+1 .
$$

We want to show that some solutions of this equation do not exist in the future.

Now

$$
\int_{1}^{\infty} \frac{d x}{1+h(x)}<\int_{1}^{\infty} \frac{d x}{1+x^{2}}+\sum_{n=2}^{\infty} \text { area } R_{n},
$$

where $R_{n}$ is the rectangle with height 1 and base $2 / n^{2}$ and center $(n, 1 / 2)$. The area of $R_{n}=2 / n^{2}$. Hence, since $\int_{1}^{\infty} d x /\left(1+x^{2}\right)<\infty$ and $\sum_{n=2}^{\infty}$ area $R_{n}<\infty$, we have $\int_{1}^{\infty} d x /(1+h(x))<\infty$. This condition on $1+h(x)$ is sufficient for there to exist solutions of $\dot{x}=1+h(x)$ which do not exist in the future.

One unusual property, that we will later consider more deeply, of the system $\dot{x}=h(x)$ is that although all solutions exist in the future, we have for any $\varepsilon>0$, not all solutions of $\dot{x}=h(x)+\varepsilon$ exist in the future.

Thus, in discussing a class of perturbation functions $\phi(r)$ for (ES), we expect that $\dot{\phi}(r)$ depends on $\gamma(r)$. In fact we will often impose the following conditions on $\phi$ and $\gamma$ :

$$
\lim \sup \dot{\phi}(r) / \gamma(r)<\infty,
$$


or

$$
\liminf _{r \rightarrow \infty} \phi(r) / \gamma(r)>0 \text {. }
$$

Moreover, by imposing a linearity or Lipschitz condition on $\gamma$ we are able to construct the corresponding admissible perturbation classes which are independent of the unperturbed system. Examples are provided to show these classes are "maximal." By assuming our perturbation term depends on $t$ we provide sufficient conditions on $\gamma(x)$ such that all solutions of $\dot{x}=\gamma(x)+g(t)$ exist in the future. For a large class of equations necessary and sufficient conditions on $\gamma(x)$ are given such that all solutions of (PS) exist in the future when all solutions of (ES) exist in the future.

\section{Perturbed differential scalar equations.}

THEOREM 3.1. Assume all solutions of (ES) exist in the future. Then all solutions of (PS) exist in the future for any $\dot{\rho}$ satisfying (2.2).

Proof. Since solutions of (ES) exist in the future then $\int^{\infty} d r / \gamma(r)=$ $\infty$. From (2.2) there exist $N$ and $K$ satisfying

$$
\phi(r) / \gamma(r)<K \text { for } r>N
$$

Therefore,

$$
\begin{aligned}
\dot{\phi}(r)+\gamma(r) & \leqq(1+K) \gamma(r), r>N \Longrightarrow 1 /(\dot{\phi}(r)+\gamma(r)) \\
& \geqq 1 /((1+K) \gamma(r)), r>N .
\end{aligned}
$$

Since $\int^{\infty} d r / \gamma(r)=\infty$,

$$
\int_{\phi}^{\infty} \frac{d r}{\phi(r)+\gamma(r)}=\infty
$$

Noting that $\lim \inf _{r \rightarrow \infty} \dot{\phi}(r) / \gamma(r)>0 \Rightarrow \lim \sup _{r \rightarrow \infty} \gamma(r) / \dot{\phi}(r)<\infty$ the following dual theorem is immediately obtained by interchanging the roles of $\gamma$ and $\phi$.

THEOREM 3.2. Assume solutions of $\dot{r}=\phi(r)$ exist in the future. Then all solutions of (PS) exist in the future for any $\gamma$ satisfying (2.3).

Corollary 3.3. Assume that $\gamma$ is bounded. If $\phi \in \bar{\Delta}$ then all solutions of (PS) exist in the future. 
Proof. The limit of $\phi(r)$ as $r \rightarrow \infty$ either exists or is equal to $\infty$. Since $\gamma(\cdot)$ is bounded there exists a $C>0$ such that $\gamma(r)<C$. Hence we have for $\dot{\phi}(r) \not \equiv 0$,

$$
\phi(r) / \gamma(r)>\phi(r) / C=\liminf _{r \rightarrow \infty} \phi(r) / \gamma(r)>\lim _{r \rightarrow \infty} \phi(r) / C>0 \text {. }
$$

The result follows from Theorem 3.2.

COROLlary 3.4. Assume solutions of $\dot{r}=\lambda(t) \gamma(r)$ exist in the future, where $\lambda$ is continuous. Then solutions of $\dot{r}=\lambda(t) \gamma(r)+\chi(t) \phi(r)$ exist in the future for $\chi(t)$ continuous, and for $\phi$ satisfying (2.2).

Proof. Suppose that solutions of

$$
\dot{r}=\lambda(r) \gamma(r)+\chi(t) \phi(r)
$$

do not exist in the future. Then there exist $r_{0}, t_{0}, \hat{t}$, and a solution $r(\cdot)$ of $(3.1)$ such that

$$
r\left(t, t_{0}, r_{0}\right) \rightarrow \infty \text { as } t \rightarrow \hat{t}, \hat{t}>t_{0} .
$$

On the compact set $\left[t_{0}, \hat{t}\right]$ there exists a $B>0, T>0$ such that $\lambda(t) \leqq B$ and $\chi(t) \leqq T$. Consider the equation

$$
\dot{s}=B \gamma(s)+T \dot{\phi}(s) \text {. }
$$

Certainly $B \gamma$ and $T \dot{\phi}$ satisfies (2.2). Hence by Theorem 3.1 all solutions of (3.2) exist in the future. In particular all solutions of (3.2) are bounded on $\left[t_{0}, \hat{t}\right]$ which implies all solutions of (3.1) are bounded on $\left[t_{0}, \hat{t}\right]$, a contradiction, thus proving the result.

A dual result using Theorem 3.2 can easily be obtained when (2.3) is satisfied.

When we observe Example 2.3 we notice that all solutions of $\dot{x}=$ $h(x)+1$ do not exist in the future. One natural question is what functions will perturb $\dot{x}=h(x)$ and preserve the extendability of solutions. We will now consider this question in a more general form. If we suppose all solutions of (ES) exist in the future, then what conditions on $\gamma$ are needed to insure that all solutions of

$$
\dot{x}=\gamma(x)+g(t),
$$

where $g: R \rightarrow R^{+}$is continuous, exist in the future? With Theorem 3.1 we prove the following result.

COROLlary 3.5. Assume all solutions of (ES) exist in the future and that either 


$$
\limsup _{r \rightarrow \infty} \gamma(r)<\infty
$$

or

$$
\liminf _{r \rightarrow \infty} \gamma(r)>0 \text { is satisfied. }
$$

Then for continuous function $g: R \rightarrow R^{+}$we have that all solutions of $(\mathrm{PT})$

$$
\dot{x}=\gamma(x)+g(t)
$$

exist in the future.

Proof. Since $g(t)=g(t) \cdot 1,(3.3)$ and (3.4) implies (2.2) and (2.3) respectively for $\phi(r) \equiv 1$. Applying Corollary 3.4 we arrive at the result.

REMARKS. It follows from Example 2.3 that we cannot improve the conditions on $\gamma$ in Corollary 3.5 since in that case $\gamma(x)=h(x)$, $g(t)=1$, and $\lim \sup _{x \rightarrow \infty} h(x)=\infty$ and $\lim \inf _{x \rightarrow \infty} h(x)=0$.

Results for systems (E) and (P) may be obtained by assuming $|f(t, x)| \leqq \gamma(|x|)$ and $|g(t, x)| \leqq \phi(|x|)$ where $\gamma(\cdot)$ and $\phi(\cdot)$ satisfy the hypotheses of the previous theorems and corollaries.

We have shown that if $\gamma$ and $\dot{\phi}$ belong to $\Delta$ then it is not necessarily true that all solutions of (PS) exist in the future unless $\gamma$ and $\phi$ satisfy (2.2) or (2.3). The problem then is to consider a restriction of the class $\bar{\Delta}$, call it $L$, such that if $\gamma \in L$, and $\phi \in \bar{\Delta}$, where $\gamma$ and $\phi$ are independent of each other, then all solutions of (PS) exist in the future.

DEFINITION 3.6. Let $L$ be the class of continuous functions $\gamma$ : $[0, \infty) \rightarrow(0, \infty)$ satisfying $|\gamma(x)| \leqq K|x|+h(t)$ where $h: R \rightarrow R^{+}$is continuous and $K$ is any positive constant.

The object of the next theorem is to prove essentially that $L+$ $\bar{\Delta} \subset \Delta$.

THEOREM 3.7. We are given (ES), where $\gamma \in L$. Then if $\phi \in \bar{\Delta}$ all solutions of (PS) exist in the future.

The solution of (PS) satisfies

$$
\dot{r} \leqq K r+\dot{\phi}(r)+g(t) .
$$

Letting $y=e^{-K t} r$, we see that (3.5) becomes

$$
\dot{y} \leqq L_{1}(t, y)+h(t),
$$

where $h(t)=e^{-K t} g(t)$ and $L_{1}(t, y)=e^{-K t}\left(\phi\left(y e^{K t}\right)\right)$. If we consider any 
interval $\left[t_{0}, T\right]$ where $t_{0}$ is fixed and $T>t_{0}$, then we have for $t \in\left[t_{0}, T\right]$

$$
L_{1}(t, y) \leqq e^{-K t_{0}}\left(\phi\left(y e^{K T}\right) \equiv L_{2}(y)\right.
$$

since $\phi$ is monotone. We also note that $\int^{\infty} d y / L_{2}(y)=\infty$ and $L_{2}(\cdot)$ is monotone, and using Corollary 3.5 we have that all solutions of

$$
\dot{y}=L_{2}(y)+h(t)
$$

exist on $\left[t_{0}, T\right]$. Since $T$ is arbitrary, the result follows.

REMARK. As we have done before, we can obtain results for (E) and $(\mathrm{P})$ by allowing $f$ and $g$ to satisfy

$$
|f(t, x)| \leqq \lambda(t) \phi(|x|)
$$

and

$$
|g(t, x)| \leqq \chi(t) \psi(|x|),
$$

in which $\lambda, \chi$ are continuous. By assuming $\phi(\cdot) \in L$ and $\psi(\cdot) \in \bar{\Delta}$, we arrive at the same results. In particular $L$ can be extended to include Lipschitz functions and linear functions.

Theorem 3.7 is a generalization of a theorem of Stokes [5]. In his theorem, however, he assumed a linearity condition on $f(t, x)$, namely, that $f(t, x)=A(t) x$, where all solutions of $\dot{x}=A(t) x$ are uniformly stable. He arrived at the same result using the Tychonoff fixed point theorem. With the general conditions assumed in Theorem 3.7, we are not able to use this method.

In a meaningful sense we shall show $\bar{\Delta}+\bar{\Delta} \not \subset \Delta$, implying that Theorem 3.7 cannot be significantly strengthened.

ExAmple 3.8. Consider the functions $\psi(\cdot)$ and $\phi(\cdot)$ where $\psi$ and $\phi$ depend upon each other as follows: we consider the initial values

$$
x_{0}=0, x_{1}=1, x_{2}=2, \psi\left(x_{0}\right)=1 \text {, and } \phi\left(x_{0}\right)=2 .
$$

For all $n \geqq 1$, we define

$$
\psi\left(x_{2 n-1}\right)=-\phi\left(x_{2 n-2}\right)+3(2)^{2 n-1}\left(x_{2 n}-x_{2 n-1}\right),
$$

and define $\psi(x)=\psi\left(x_{2 n-1}\right)$ for all $x$ satisfying

$$
x_{2 n-1} \leqq x \leqq x_{2 n+1},
$$

where $x_{2 n+1}=x_{2 n-1}+\psi\left(x_{2 n-1}\right)$. Similarly, we define

$$
\phi\left(x_{2 n}\right)=-\psi\left(x_{2 n-1}\right)+3(2)^{2 n}\left(x_{2 n+1}-x_{2 n}\right),
$$


and define $\phi(x)=\phi\left(x_{2 n}\right)$ for all $x$ satisfying

$$
x_{2 n} \leqq x \leqq x_{2 n+2},
$$

where $x_{2 n+2}=x_{2 n}+\phi\left(x_{2 n}\right)$. With these initial values we can generate all the points $x_{n}$, the constants $\psi\left(x_{2 n-1}\right), \phi\left(x_{2 n}\right)$, and hence we can then define $\phi(x)$ and $\psi(x)$ inductively as step functions on $R^{+}$.

By their constructions, we have

$$
\int_{0}^{\infty} \frac{d x}{\phi(x)}=\sum_{n=1}^{\infty} 1=\infty, \text { and } \int_{0}^{\infty} \frac{d x}{\psi(x)}=\sum_{n=1}^{\infty} 1=\infty
$$

Since $\phi(\cdot)$ and $\psi(\cdot)$ are monotone, we have that they are each in $\bar{\Delta}$.

We claim that $\phi+\psi \not \subset \Delta$. To show this it is sufficient to show

$$
\int_{0}^{\infty} \frac{d x}{\phi(x)+\psi(x)}<\infty
$$

We see that

$$
\begin{aligned}
\int_{0}^{\infty} \frac{d x}{\phi(x)+\psi(x)} & =1 / 3+1 / 6+1 / 12+\cdots \\
& =(1 / 3)\left(1+1 / 2+1 / 2^{2}+\cdots\right)=(1 / 3) \sum_{n=0}^{\infty} 1 / 2^{n}<\infty,
\end{aligned}
$$

thus proving the result. Hence the solution of

$$
\dot{x}=\phi(x)+\psi(x),
$$

with an initial condition $x\left(t_{0}\right)=x_{0}>0$, does not exist on $\left[t_{0}, \infty\right)$.

4. Necessary and sufficient conditions for extendability. From previous remarks we have observed some peculiar characteristics of Example 2.3. We shall characterize the class of continuous functions $h(x), h: R^{+} \rightarrow R^{+}$, so as to determine what properties of $h(x)$ cause solutions of

$$
\dot{x}=h(x)+K, K \text { any constant, }
$$

not to exist in the future, even though all solutions of

$$
\dot{x}=h(x)
$$

do exist in the future. The class of perturbing functions will, in fact, include the constant functions as a proper subset.

THEOREM 4.1. We assume that all solutions of

$$
\dot{x}=h(x),
$$


where $h: R^{+} \rightarrow R^{+}$is continuous, exist in the future. Then for any $\phi:[0, \infty) \rightarrow(0, \infty)$, with lim $\sup _{r \rightarrow \infty} \phi(r)<\infty$ and $\lim _{i n f} \operatorname{in}_{r \rightarrow \infty} \phi(r)>0$ some solution of

$$
\dot{x}=h(x)+\phi(x)
$$

does not exist in the future if and only if $h$ has the following properties:

$$
\text { if we let } \begin{array}{r}
S(K)=\{x: h(x) \leqq K\}, \text { then } \\
m(S(K))<\infty \text { for all } K>0,
\end{array}
$$

where $m(S(K))$ represents the Lebesgue measure of $S(K)$, and (4.2) for all $K>0$,

$$
\int_{\sim S(K)} \frac{d x}{h(x)}<\infty
$$

where $\sim S(K)$ is the complement of $S(K)$. (Note: (4.1) and (4.2) imply $\lim \sup _{x \rightarrow \infty} h(x)=\infty$ and $\liminf \operatorname{in}_{x \rightarrow \infty} h(x)=0$.)

Proof. Let (4.1) and (4.2) hold. It is sufficient to show there exists an $x_{0}>0$ such that

$$
\int_{x_{0}}^{\infty} \frac{d x}{h(x)+\phi(x)}<\infty
$$

From the hypotheses on $\dot{\phi}(x)$ there exist $K>0, x_{0}>0$ such that $\phi(x) \geqq K$ for $x \geqq x_{0}$. Hence

$$
\int_{x_{0}}^{\infty} \frac{d x}{h(x)+\dot{\phi}(x)} \leqq \int_{x_{0}}^{\infty} \frac{d x}{h(x)+K} \leqq \int_{S(K)} \frac{d x}{h(x)+K}+\int_{\sim(S(K)} \frac{d x}{h(x)+K} ;
$$

but since $h(x)+K>h(x)$, we see that from (4.2)

$$
\int_{\sim S(K)} \frac{d x}{h(x)+K}<\int_{\sim S(K)} \frac{h(x)}{d x}<\infty .
$$

Moreover, since $1 /(h(x)+K)<1 / K$, then

$$
\int_{S(K)} \frac{d x}{h(x)+K}<\int_{S(K)} 1 / K=(1 / K) m(S(K))<\infty,
$$

by (4.1), thus proving the first part.

Conversely, assume some solution of $\dot{x}=h(x)+\phi(x)$ fails to exist in the future. Assume (4.1) does not hold; that is, there exists $C>0$ such that $m(S(C))=\infty$. Since $S(C)=\{x: h(x)+\varepsilon \leqq C+\varepsilon\}$, $\varepsilon>0$, we have that 


$$
1 /(h(x)+\varepsilon) \geqq 1 /(C+\varepsilon) \text { for } x \in S(C) \text { and all } \varepsilon>0 .
$$

From the hypotheses on $\phi(x)$ there exists $K_{1}>0$ such that $\phi(x)<K_{1}$ for all $x$. Hence for $x \in S(C)$

$$
\frac{1}{h(x)+\dot{\phi}(x)} \geqq \frac{1}{C+K_{1}} .
$$

Since some solution of

$$
\dot{x}=h(x)+\phi(x)
$$

does not exist in future, there exists an $r_{0}>0$ such that

$$
\int_{r_{0}}^{\infty} \frac{d x}{h(x)+\phi(x)}<\infty
$$

If we consider the set

$$
S_{0}(C)=S(C) \backslash\left(S(C) \cap\left[0, r_{0}\right]\right),
$$

then

$$
m\left(S_{0}(C)\right)=m(S(C))-m\left(S(C) \cap\left[0, r_{0}\right]\right) \geqq m(s(C))-r_{0}=\infty .
$$

Therefore,

$$
\int_{r_{0}}^{\infty} \frac{1}{h(x)+\phi(x)} \geqq \int_{S_{0}(C)} \frac{d x}{C+K_{1}}=\frac{1}{C+K_{1}} m\left(S_{0}(C)\right)=\infty,
$$

a contradiction.

If we now assume (4.2) does not hold, then there exists $K>0$ such that

$$
\int_{\sim S(K)} \frac{d x}{h(x)}=\infty
$$

For $x \in \sim(S(K))$ we have $h(x)>K$, which implies $h(x)+K<2 h(x)$. Hence,

$$
\begin{aligned}
\frac{1}{2} \int_{\sim S(K)} \frac{d x}{h(x)} & <\int_{\sim S(K)} \frac{d x}{h(x)+K} \\
& \leqq \int_{r_{0}}^{\infty} \frac{d x}{h(x)+K}+\int_{0}^{r_{0}} \frac{d x}{h(x)+K} \leqq \int_{r_{0}}^{\infty} \frac{d x}{h(x)+K}+r_{0} / K .
\end{aligned}
$$

Since

$$
\int_{r_{0}}^{\infty} \frac{d x}{h(x)+K}=\infty \text { implies } \int_{r_{0}}^{\infty} \frac{d x}{h(x)+C}=\infty
$$

for all $C>0$, a contradiction arises for $C=K_{1}$, hence proving the 
result.

An immediate corollary characterizes those $h(x)$ such that all solutions of $\dot{x}=h(x)+\phi(x)$ exist in the future.

CoRollary 4.2. Suppose all solutions of $\dot{x}=h(x)$, where $\left.h: R^{+} \rightarrow R^{+}\right)$, is continuous, exist in the future. Then for each $\phi:[0, \infty) \rightarrow(0, \infty)$ with $\lim _{r \rightarrow \infty} \sup \phi(r)<\infty, \lim _{r \rightarrow \infty} \inf \phi(r)>0$, all solutions of $\dot{x}=h(x)+$ $\phi(x)$ exist in the future if and only if $h(x)$ has one of the following two properties.

(4.3) there exists $C>0$ such that $m(S(C))=\infty$,

or

$$
\int_{\sim S(K)} \frac{d x}{h(x)}=\infty \text { for some } K>0
$$

We thus see it is possible to investigate the extendability of solutions of $\dot{x}=h(x)+\phi(x)$ by analyzing $h(x)$ on a possibly "small" subset of $[0, \infty)$.

5. Applications to boundedness and uniqueness. The preceding results naturally extend to the case when solutions are uniformly bounded. In particular, it is well known that if $|f(t, x)| \leqq \lambda(t) \psi(|x|)$, where $\psi \in \Delta$ and $\int_{0}^{\infty} \lambda(t) d t<\infty$, then solutions of $(\mathrm{E})$ are uniformly bounded. Moreover if $\left|g^{\prime}(t, x)\right| \leqq \mu(t) \phi(|x|)$, where $\int_{0}^{\infty} \mu(t) d t<\infty, \dot{\phi} \in \Delta$, and $\phi$ and $i$ satisfy either (2.2) or (2.3) then all solutions of $(\mathrm{P})$ are uniformly bounded. This follows from the fact that solutions of

$$
\dot{r}=(\lambda(t)+\mu(t))(\psi(r)+\dot{\phi}(r))
$$

are uniformly bounded since $\int_{0}^{\infty}(\lambda(t)+\mu(t)) d t<\infty$ and $(\psi(r)+\phi(r)) \in \Delta$ (Theorems 3.1 and 3.2). In a similar manner we can extend our other results to obtain analogous conclusions about perturbed uniform bounded systems.

Our results also extend to questions of uniqueness by applying the Osgood criterion [2, p. 33]: namely, the only solution $u(t)$ of the differential equation

$$
u^{\prime}=a(t) \gamma(u)
$$

where $a(t) \geqq 0$ is continuous, $\psi(u)$ is continuous for $u \geqq 0, \psi(0)=0$, $\psi(u)>0$ if $u>0$, and $\int_{+0} d u / \psi(u)=\infty$, on any interval $(0,0+\varepsilon]$ satisfying $u(0)=0$, is $u(t) \stackrel{+0}{\equiv} 0$. Applying our previous results we obtain 
the following uniqueness theorem on perturbed systems: assume the previous conditions on (A). Then for any continuous function $b(t)$ and for any function $\phi(u)$ satisfying $\phi(0)=0, \phi(u)>0$ if $u>0$, and

$$
\limsup _{r \rightarrow 0} \dot{\phi}(r) / \gamma(r)<\infty,
$$

the only solution of

$$
u^{\prime}=a(t) \gamma(r)+b(t) \phi(r)
$$

on $(0, \varepsilon]$ satisfying $u(0)=0$ is $u(t) \equiv 0$. Applying Kamke's Theorem [2, p. 31], results for systems $(\mathrm{E})$ and $(\mathrm{P})$ may also be obtained. Similarly some of our other results can be extended to obtain information concerning the uniqueness of solutions of perturbed systems.

\section{REFERENCES}

1. R. Conti, Sulla prolungabilita delle soluzioni di un sistema di equazioni differenziali ordinaire, Boll. Un. Mat. Ital., 11 (1956), 510-514.

2. P. Hartman, Ordinary Differential Equations, Wiley and Sons, New York, 1964.

3. V. Lakshmikantham and S. Leela, Differential and Integral Inequalities, Volume I, Academic Press, New York, 1969.

4. J. LaSalle and S. Lefschetz, Stability by Liapunov's Direct Method with Applications, Academic Press, New York, 1961.

5. A. Stokes, The application of a fixed point theorem to a variety of non-linear stability problems, Contrib. to the Theory of Non-Linear Oscillations, 5 (1960), 173-184. 6. T. Yoshizawa, Stability Theory by Liapunov's Second Method, The Mathematical Society of Japan, Tokyo, 1966.

Received May 18, 1971. This work is part of the author's doctoral thesis under the direction of Prof. A. Strauss at the University of Maryland.

UNIVERSITY OF MisSOURI-Columbia 


\section{PACIFIC JOURNAL OF MATHEMATICS}

\section{EDITORS}

\author{
H. SAMELSON \\ Stanford University \\ Stanford, California 94305 \\ C. R. HOBBY \\ University of Washington \\ Seattle, Washington 98105
}

\section{J. DuGundJI}

Department of Mathematics University of Southern California Los Angeles, California 90007

RICHARD ARENS

University of California

Los Angeles, California 90024

\section{ASSOCIATE EDITORS}
E. F. BECKENBACH
B. H. NeumanN
F. WOLF
K. YOSHIDA

\section{SUPPORTING INSTITUTIONS}

\author{
UNIVERSITY OF BRITISH COLUMBIA \\ CALIFORNIA INSTITUTE OF TECHNOLOGY \\ UNIVERSITY OF CALIFORNIA \\ MONTANA STATE UNIVERSITY \\ UNIVERSITY OF NEVADA \\ NEW MEXICO STATE UNIVERSITY \\ OREGON STATE UNIVERSITY \\ UNIVERSITY OF OREGON \\ OSAKA UNIVERSITY
}

\author{
UNIVERSITY OF SOUTHERN CALIFORNIA \\ STANFORD UNIVERSITY \\ UNIVERSITY OF TOKYO \\ UNIVERSITY OF UTAH \\ WASHINGTON STATE UNIVERSITY \\ UNIVERSITY OF WASHINGTON \\ AMERICAN MATHEMATICAL SOCIETY \\ NAVAL WEAPONS CENTER
}

The Supporting Institutions listed above contribute to the cost of publication of this Journal, but they are not owners or publishers and have no responsibility for its content or policies.

Mathematical papers intended for publication in the Pacific Journal of Mathematics should be in typed form or offset-reproduced, (not dittoed), double spaced with large margins. Underline Greek letters in red, German in green, and script in blue. The first paragraph or two must be capable of being used separately as a synopsis of the entire paper. The editorial "we" must not be used in the synopsis, and items of the bibliography should not be cited there unless absolutely necessary, in which case they must be identified by author and Journal, rather than by item number. Manuscripts, in duplicate if possible, may be sent to any one of the four editors. Please classify according to the scheme of Math. Rev. Index to Vol. 39. All other communications to the editors should be addressed to the managing editor, Richard Arens, University of California, Los Angeles, California, 90024.

50 reprints are provided free for each article; additional copies may be obtained at cost in multiples of 50 .

The Pacific Journal of Mathematics is published monthly. Effective with Volume 16 the price per volume (3 numbers) is $\$ 8.00$; single issues, $\$ 3.00$. Special price for current issues to individual faculty members of supporting institutions and to individual members of the American Mathematical Society: $\$ 4.00$ per volume; single issues $\$ 1.50$. Back numbers are available.

Subscriptions, orders for back numbers, and changes of address should be sent to Pacific Journal of Mathematics, 103 Highland Boulevard, Berkeley, California, 94708.

PUBLISHED BY PACIFIC JOURNAL OF MATHEMATICS, A NON-PROFIT CORPORATION

Printed at Kokusai Bunken Insatsusha (International Academic Printing Co., Ltd.), 270, 3-chome Totsuka-cho, Shinjuku-ku, Tokyo 160, Japan. 


\section{Pacific Journal of Mathematics}

\section{Vol. 42, No. $2 \quad$ February, 1972}

Stephen Richard Bernfeld, The extendability of solutions of perturbed scalar differential equations ................................. 277

James Edwin Brink, Inequalities involving $f_{-} p$ and $f^{(n)}{ }_{q}$ for $f$ with $n$

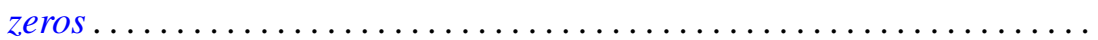

Orrin Frink and Robert S. Smith, On the distributivity of the lattice of filters of a groupoid

Donald Goldsmith, On the density of certain cohesive basic sequences .... 323

Charles Lemuel Hagopian, Planar images of decomposable continua . . . . . 329

W. N. Hudson, A decomposition theorem for biadditive processes ........ 333

W. N. Hudson, Continuity of sample functions of biadditive processes......

Masako Izumi and Shin-ichi Izumi, Integrability of trigonometric series.

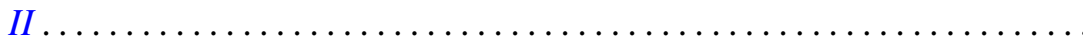

H. M. Ko, Fixed point theorems for point-to-set mappings and the set of

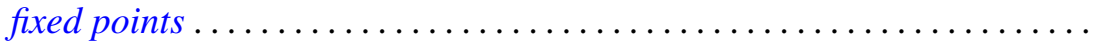

Gregers Louis Krabbe, An algebra of generalized functions on an open interval: two-sided operational calculus ...

Thomas Latimer Kriete, III, Complete non-selfadjointness of almost selfadjoint operators.................................

Shiva Narain Lal and Siya Ram, On the absolute Hausdorff summability of a Fourier series .

Ronald Leslie Lipsman, Representation theory of almost connected groups...

James R. McLaughlin, Integrated orthonormal series.... . .

H. Minc, On permanents of circulants.

Akihiro Okuyama, On a generalization of $\Sigma$-spaces.....

Norberto Salinas, Invariant subspaces and operators of class $(S)$

James D. Stafney, The spectrum of certain lower triangular matrices as operators on the $l_{p}$ spaces .......................

Arne Stray, Interpolation by analytic functions

$\mathrm{Li} \mathrm{Pi} \mathrm{Su}$, Rings of analytic functions on any subset of the complex plane.

R. J. Tondra, A property of manifolds compactly equivalent to compact manifolds.... 